\title{
The Role of Circadian Regulation in Cancer
}

\author{
S. GeRY AND H.P. KoEFFLeR \\ Cedars-Sinai Medical Center, Division of Hematology/Oncology, University of California
}

School of Medicine, Los Angeles, California 90048

\begin{abstract}
Proper circadian regulation is essential for the well being of the organism, and disruption of circadian rhythms is associated with pathological conditions including cancer. In mammals, the core clock genes, Per 1 and Per2, are key regulators of circadian rhythms both in the central clock in the hypothalamous and in peripheral tissues. Recent findings revealed molecular links between Per genes and cellular components that control fundamental cellular processes such as cell division and DNA damage. New data also shed light on mechanisms by which circadian oscillators operate in peripheral organs to influence tissuedependent metabolic and hormonal pathways. Circadian cycles are linked to basic cellular functions, as well as to tissue-specific processes through the control of gene expression and protein interactions. By controlling global networks such as chromatin remolding and protein families, which themselves regulate a broad range of cellular functions, circadian regulation impinges upon almost all major physiological pathways. These molecular insights illustrate how disregulation of circadian rhythms might influence the susceptibility to cancer development and provide further support for the emerging role of circadian genes in tumor suppression.
\end{abstract}

\section{INTRODUCTION}

Most living organisms exhibit behavioral and physiological circadian rhythms, allowing them to adapt to the daily cycle of light and dark. In mammals, key physiological processes, including sleep-wake cycles, glucose and lipid metabolism, hormone secretion, blood pressure, DNA-damage response, immunity, and cell cycle, are influenced by circadian rhythms. Furthermore, perturbations of these rhythms both in humans (as occurs in night shift workers) and animal experimental models have been associated with diverse pathogenic conditions, such as sleep disorders, depression, diabetes, obesity, and malignant transformation.

Circadian rhythms are driven by a master clock located in the hypothalamic suprachiasmatic nucleus $(\mathrm{SCN})$ that synchronizes numerous subsidiary oscillators in peripheral tissues. The circadian clockwork in both the SCN and the peripheral cells is regulated by finely tuned transcription-translation feedback loops and posttranslational modifications that are maintained by a core set of clock genes (Shearman et al. 2000; Reppert and Weaver 2002; Schibler and Sassone-Corsi 2002; Ko and Takahashi 2006). The positive feedback loop involves two transcription factors, Clock and Bmall which dimerize and bind to E-boxes in the promoters of a large number of target genes. These include their own negative regulators Period (Per1, 2, and 3 ) and cryptochrome (Cryl and Cry2). During the course of the day, Per and Cry proteins accumulate and multimerize in the cytoplasm. They then translocate to the nucleus in a phosphorylation-dependent manner where they repress the Clock-Bmall complex, thus forming the major negative circadian feedback loop. For a new cycle to begin, the inhibitory proteins must be removed. Per and Cry are phosphorylated and degraded, releasing the repression of the Clock-Bmall transcriptional activity. An additional feedback loop involves the orphan nuclear receptors Rev-erbo and Rora that, respectively, activate and repress Bmall expression. This adds stability and robustness to the clock, reinforcing the oscillations.

The same molecular mechanisms that regulate core clock components in the SCN, i.e., transcription-translation feedback loops and posttranslational modifications, are used by the clock to regulate various genes in peripheral tissues, such as throughout the body, fundamental cellular pathways like the cell cycle and metabolic cycles are under circadian regulation (Lowrey and Takahashi 2004). Expression profiling studies demonstrated that in any given tissue, up to $10 \%$ of transcripts are clock-controlled genes exhibiting circadian oscillation (Panda et al. 2002; Storch et al. 2002). The Clock-Bmall complex directly controls the expression of some of these genes, whereas others are indirectly regulated via circadian expression of relevant transcription factors. Significantly, some of these genes are key players in cell division, proliferation, and survival. With the realization of the importance of circadian regulation to the overall health of the organism, it becomes apparent why disruption of circadian rhythms may increase the risk for cancer. Recent findings are now beginning to shed light on the molecular mechanisms underlying the circadian-cancer connection.

\section{THE CELL CYCLE}

At a first glance, circadian rhythms, regulated by the earth's movement around the sun, and the cell division occurring within the confined boundaries of cells may not seem like obvious partners. However, a closer look reveals that these two systems have some intriguing similarities. Both the core circadian network and the cell cycle machinery are found in most cells, even in established cell lines. Both are intracellular systems that rely on phases of transcription-translation and protein modification-degradation. Another interesting finding is that like the 24-hour periodicity of biological clocks, mammalian cell division is approximately 1 day, and even in culture, most eukaryotic cells undergo division within 24 
hours or so. Circadian gene expression in fibroblasts continues during cell division, and the circadian oscillator gates cytokinesis to defined time windows; in turn, mitosis can phase-shift the circadian cycle (Nagoshi et al. 2004; Reddy et al. 2005; Hunt and Sassone-Corsi 2007). Another instructive observation is that among the hundreds of proteins exhibiting circadian expression patterns, several are key regulators of the cell cycle and apoptosis. For example, c-Myc, a well-known cell cycle transcription factor, and Wee-1, a cell cycle checkpoint kinase, contain E-boxes in their promoters and are under direct transcriptional regulation by the Clock-Bmall complex (Fu et al. 2002; Matsuo et al. 2003). The levels of the apoptotic proteins BCL2 and BAX rhythmically oscillate in murine bone marrow (Granda et al. 2005).

Studies in mice with mutations in core clock genes clearly demonstrate the critical role for proper circadian regulation in cell cycle progression in peripheral tissues. After partial hepatectomy, livers from cry-deficient mice regenerate slower than those from normal mice. In this model, the circadian clock was shown to control the $G_{2} / M$ transition by regulating the expression of wee1, which negatively regulates the $\mathrm{Cdc} 2-$ cyclin $\mathrm{B} 1$ complex, the major cyclin complex governing $\mathrm{G}_{2} / \mathrm{M}$ (Matsuo et al. 2003). Per 2 mutant mice have increased susceptibility to both spontaneous and radiation-induced tumor development. These mutant mice show aberrant temporal expression of cell cycle genes such as $p 53$, c-myc cyclin $A$, and $m d m 2$, leading to deregulated cell division and reduced levels of apoptosis (Fu et al. 2002). In clock mutant mice, several cell cycle inhibitory genes are up-regulated, e.g., $p 21^{\text {(WAF1) }}, p 27^{(\mathrm{Kip} 1)}, \operatorname{chk} 1, \operatorname{chk} 2$, and $a t r 1$, whereas proproliferative genes such as jak2, ER $\alpha$, akt1, cdk2, cyclins $D 3$ and $E 1$, and the transforming growth factor- $\beta$ (TGF$\beta$ ) and epiderminal growth factor (EGF) receptors are down-regulated. Fibroblasts derived from clock mutant embryos exhibit reduced DNA synthesis and cell proliferation compared with normal fibroblasts (Miller et al. 2007). We and other investigators have shown that ectopic expression of Per 1 and Per 2 in human cancer cell lines results in deregulated expression of cell cycle genes such as c-myc, p21, wee1, cyclin B1, and $c d c 2$, associated with cell cycle arrest and apoptosis (Gery et al. 2005, 2006, 2007a,b; ; Hua et al. 2006). These data establish a strong link between the circadian clock and the cell cycle. Furthermore, they may explain how disruption of the clock may shift the cellular balance between proproliferative versus antiproliferative genes, increasing the risk for caner initiation and progression.

\section{DNA DAMAGE}

For early life forms, strong UV irradiation during the day must have been a constant source of DNA damage; hence, restricting the $\mathrm{S}$ phase of the cell cycle to nighttime may have had an adaptive value (Woelfle et al. 2004; Reddy et al. 2005). In current multicellular organisms as humans and other mammals, DNA damage leading to genomic instability is a major force driving cancer. To cope with this, cells have developed a network of checkpoint pathways allowing them to initiate either cell cycle arrest and DNA repair or apoptosis. Recent findings show that the circadian system impinges on some of the key players within these networks. Moreover, the relationship between the circadian clock and the DNA-damage response appears to persevere across species, as it is found in diverse organisms from plants to mammals. An early interesting clue on how clock genes may influence the cellular DNA-damage response came from the observation that the expression of Per 1, Per 2, Clock, Cryl, and Bmall is induced in the liver of mice that undergo exposure to $\gamma$-radiation. This induction is absent in Per2 mutant mice, suggesting a role of Per2 in cellular pathways that govern the response to stress. The Per2-deficient mice are more sensitive to $\gamma$-radiation, showing premature hair graying, hair loss, and high frequency of development of lymphoma, attributed, at least partially, to decreased apoptosis in damaged cells. These findings raised the possibility that by regulating the DNA-damage response, Per 2 functions in tumor suppression (Fu et al. 2002; Lee 2006). Our studies focused on Perl and have shown that Perl has an important role in coupling the circadian cycle to the DNA-damage response and to growth control. In human cancer cells, $\gamma$-radiation induces Per 1 expression and nuclear localization. Overexpression of Perl sensitizes these cells to DNA-damage-induced apoptosis, whereas its inhibition has the opposite effect. This phenotype is associated with altered expression of cMyc and p21 (Gery et al. 2006). Deregulated expression of c-myc was suggested to be a key factor leading to tumor development in Per 2 mutant mice. In addition, malignant growth in mice with disruption of circadian coordination has been suggested to result from abnormal c-myc expression (Filipski et al. 2006).

An even more intimate relationship between the circadian clock and the DNA-damage response was recently revealed by several groups who demonstrated that core clock proteins directly bind to major checkpoint proteins. The Atm kinase and its downstream effector Chk2 are activated by DNA double-strand breaks and phosphorylate target proteins that initiate either DNA repair and cell cycle arrest or apoptosis (Shiloh 2003). We have shown that in human colon cancer cells, endogenous Per1 interacts with endogenous Atm and Chk2. Furthermore, ectopic expression of Per1 is sufficient to induce phosphorylation of Chk2 in the absence of DNA damage, whereas silencing of Per1 expression impairs Chk2 phosphorylation, suggesting that Per1 has a role in the DNAdamage response by interacting with and activating the Atm checkpoint pathway (Gery et al. 2006). Parallel to our findings in mammalian cells, a recent study in the fungus Neurospora demonstrated that the kinase prd-4, an ortholog of mammalian Chk2, interacts with a central clock factor in Neurospora, FRQ, and this interaction induces FRQ phosphorylation. prd-4 is itself a clock-controlled gene, suggesting a regulatory loop coupling the circadian clock and the response to DNA damage. Even more so, it was demonstrated that the DNA-damaging agent methylmethane sulfonate (MMS) can act as strong clock-resetting signal in a manner that depends on the time of day and that prd-4 is essential to resetting the clock (Pregueiro et al. 2006). 


\section{CIRCADIAN REGULATION IN CANCER}

Anther clock gene that has been shown to interact directly with checkpoint proteins is Timeless. This protein is essential for circadian rhythms in Drosophila and is required for the maintenance of robust circadian rhythms in mice. Human Timeless sustains the DNA replication fork movement in the absence of damage, whereas its partner Tipin slows DNA replication in UV-damaged cells. Timeless was found to be in a complex that includes the Atm-related kinase Atr and Atrip, a substrate of ATR. In addition, down-regulation of Timeless in human cells compromises the S-phase checkpoint, demonstrating that a clock gene coordinates cell cycle progression thought the S-phase checkpoint in response to DNA damage (Unsal-Kacmaz et al. 2005, 2007; Yoshizawa-Sugata and Masai 2007). hClk2 is the human homolog of the Caenorhabditis elegans biological clock protein, CLK-2. A recent study demonstrated that hClk2 associates with a number of checkpoint proteins including Atr and Chk1. hClk2 promotes activation of the S-phase checkpoint and downstream DNA repair by preventing unscheduled Chk1 degradation by the proteasome (Collis et al. 2007). Together, these findings provide a molecular mechanism demonstrating how the circadian clock functions in the peripheral tissues to control the cell cycle, apoptosis, and malignant growth. An emerging theme from these studies is that both transcriptional regulation and direct proteinprotein interactions couple the clock and the DNA-damage response in an evolutionary conserved manner. This suggests that these ancient systems may have evolved parallel to each other, helping cells make critical cell cycle and apoptotic decisions and, in doing so, contributing to the long-term survival of the organism.

\section{CHROMATIN}

Transcriptional activation and repression usually involves chromatin modifications such as acetylation and methylation. Chromatin changes are therefore an important normal cellular function, yet alterations in chromatin patterns occur under pathogenic conditions, including cancer (Jones and Baylin 2007). Human tumors show a massive overall loss of DNA methylation, whereas certain promoters acquire specific hypermethylation. Patterns of histone acetylation also display changes during cancer development. One consequence of these alterations is the silencing of tumor suppressor genes, owing to aberrant methylation of $\mathrm{CpG}$ islands and deacetylation of histones in their promoter regions. Epigenetic interventions, particularly those targeting histone deacetylase (HDAC), are among the most promising therapies for cancer, and HDAC inhibitors are already being used in the clinic. Moreover, because epigenetic changes occur early in tumorgenesis and are associated with distinctive cancer types, they could represent targets for chemoprevention and early diagnosis.

Finely tuned transcriptional regulation is at the heart of the circadian clock, and with approximately $10 \%$ of mammalian transcripts showing daily oscillations, it is clear that genome-wide architecture is required for proper maintenance of the clock transcriptional machinery. It was therefore hypothesized that chromatin remodeling may be a key element in the clock transcriptional regulation.
Several recent exciting discoveries demonstrate that this indeed is the case. The finding that changes in histone modifications are an important step in light-induced gene expression in neurons of the hypothalamic SCN provided early evidence that dynamic chromatin remodeling occurs within the clock system (Crosio et al. 2000). Later studies showed that the cyclic binding and release of the CLOCKBMAL1 activator complex to E boxes correlates with rhythmic changes in acetylation and methylation of surrounding DNA of target genes. Specifically, histone H3 is acetylated in chromatin that encompasses the promoters of Per1, Per2, Cryl, and the clock output gene, Dbp, when these genes are actively transcribed (Etchegaray et al. 2003; Curtis et al. 2004; Naruse et al. 2004; Ripperger and Schibler 2006). As histone acetylation promotes transcription, enzymes that acetylate histones, histone acetyltransferases (HATs), act as transcriptional coactivators by interactions with sequence-specific DNA-binding transcription factors. HAT activity, is in fact, required for Clock-Bmal1-dependent trans-activation, and as it turns out, Clock itself displays HAT activity with specificity to histones H3 and H4 (Doi et al. 2006).

Abnormal expression of circadian genes has been reported in human tumors including breast, pancreas, and endometrial cancers, as well as in hematological malignancies (Chen et al. 2005; Gery et al. 2005, 2006, 2007b; Yeh et al. 2005; Pogue-Geile et al. 2006; M.Y. Yang et al. 2006). In a number of studies, clock gene deregulation was associated with promoter hypermethylation. In a study of 55 Taiwanese women, disturbances in the expression of Per1 and Per2 was found in most (>95\%) breast cancer samples, in comparison with nearby noncancerous cells. The Per gene deregulation was not caused by genetic mutations but was probably due to methylation of the Per1 or Per2 promoters (Chen et al. 2005). Expression levels of Perl were significantly decreased in endometrial carcinoma, partly due to DNA methylation of Per1 promoter (Yeh et al. 2005). The expression levels of Per1, Per2, Per3, Cry1, Cry2, and Bmal1 were significantly impaired in both chronic phase and blast crisis of chronic myeloid leukemia (CML) samples compared with those in normal samples. Although no mutations were found within the coding region of Per3, the $\mathrm{CpG}$ islands in its promoter were methylated in all the CML samples. Likewise, the $\mathrm{CpG}$ islands of Per2 were also methylated in $40 \%$ of cases (M.Y. Yang et al. 2006). Using microarray analysis, we identified Per1 as a candidate tumor suppressor, epigenetically silenced in nonsmall-cell lung cancer (NSCLC). Per1 levels were low in a large panel of NSCLC patient samples and in NSCLC cell lines compared to normal lung tissue. The down-regulation of Per1 expression was associated with hypermethylation of the Per1 promoter. Furthermore, we found that aberrant acetylation of Per1 promoter is also a potential mechanism for silencing Per1 in cancer. In cancer cell lines from various tissues including lung, colon, and breast, Per1 expression could be induced by the histone deacetylase inhibitor, SAHA. In addition, SAHA increased acetylated histone $\mathrm{H} 3$ binding to the Per1 promoter in NSCLC cells (Gery et al. 2007b). The HAT function of Clock, essential for normal transcriptional 
regulation of clock genes like Per1, may be compromised by disruption of circadian rhythms. This in turn could lead to aberrant expression of clock genes, leaving cells more prone to the development of cancer.

Mutations in clock genes have been described in humans disorders (such as familial advanced sleep phase syndrome); thus far, they have not been associated with cancer. However, clock genes are down-regulated in many human tumors, and this has been attributed at least partly to aberrant methylation and acetylation of their promoters. Rhythmic chromatin changes in the promoters of clock genes are a normal part of the circadian transcriptional control. Variation in this process could disrupt not only the expression of core clock factors, but also the web of genes and cellular pathways that are under circadian control. Chromatin remodeling by the circadian system may have a role in the global epigenetic events occurring during carcinogenesis.

\section{HORMONES}

The secretion of many endocrine and metabolic hormones is controlled by the day/night cycle, and reciprocally circulating levels of hormones influence circadian rhythms. The pineal hormone melatonin, the main hormone relaying the circadian rhythm to the peripheral organs, is another clock component tying the circadian system to cancer. Melatonin appears to suppress tumor growth in animals and has been implicated in the pathogenesis of various human cancers, in particular, breast, colon, lung, melanoma, and leukemia. This protein is secreted by the pineal gland in the brain only in darkness.

Exposure to light at night, even briefly, decreases its production, and it has been suggested that lower levels of melatonin in the serum may contribute to tumor development (Ravindra et al. 2006). Interestingly, epidemiological studies have found a positive association between breast cancer incidence and overnight shift work (Hansen 2001). An increased worldwide incidence of breast cancer is hypothesized to be related to exposure to artificial light at night and suppression of melatonin secretion (Stevens 2005). Similarly, melatonin was reported to be lower in men with prostate cancer compared with healthy men (Bartsch et al. 1985). Although the molecular mechanism underlying these clock-cancer connections remain to be determined, data have suggested that reduced melatonin levels may increase hormone-related cancer risk by affecting the production or function of the gonadal steroid hormones estrogen and androgen, as well as their receptors.

The estrous cycle is an endocrine clock orchestrated by circulating levels of the ovarian steroids, estrogen and progesterone. As with other homeostatic processes, circadian regulation has a prominent role in this endocrine cycle. In the mammary gland, one of the target tissues of ovarian hormones, estrogen is essential for normal development and physiological functions, but it is also a potent mitogen (Yager and Davidson 2006). Thus, circadian regulation may be implicated not only in estrogen homeostasis, but also in hormone-related tumorgenesis in breast epithelia cells. The biological actions of estrogens are meditated mainly by the nuclear receptors, estrogen receptor $\alpha$ and $\beta$
(ER $\alpha$ and $E R \beta)$, which bind to estrogen response elements in the regulatory regions of target genes and associate with basal transcription factors and tissue-specific cofactors to alter gene expression. Extensive studies have identified numerous proteins and processes involved in the ER signal transduction pathway (McDonnell and Norris 2002). Given that transcriptional regulation is a key feature of both the circadian machinery and the ER pathway and the proposed connections between the clock and breast cancer, we hypothesized that the ER signaling is yet another front on which core clock factors may exert their effects. Recently, we found that Per2 is a novel estrogen-inducible ER $\alpha$ cofactor. Per2 interacts with ER $\alpha$, enhances receptor degradation, and suppresses estrogen-mediated transcription of ER target genes in breast cancer cells. In turn, Per2 itself is estrogen-inducible in these cells. Our results suggest a feedback mechanism to attenuate estrogen response that couples the circadian clock to the estrogen pathway. Overexpression of Per2 in breast cancer cell lines leads to significant growth inhibition, proliferation arrest, and apoptosis associated with deregulated expression of c-Myc, Cyclin D1, and p21, indicating that Per2 may function as a tumor suppressor in breast tissue (Gery et al. 2007a). Interestingly, Per2 was also suggested to have a role in normal mammary cell differentiation (Metz et al. 2006). Studies in rats have shown that estrogen differentially regulates the expression of per 1 and per 2 in specific brain regions as well as in some peripheral tissues (i.e., liver, kidney, and uterus) (Nakamura et al. 2005; Perrin et al. 2006). A circadian clock was reported to function in rat ovarian cells, further implicating circadian regulation in local endocrine tissues, as well as its role in the hypothalamopituitary-ovarian axis (Fahrenkrug et al. 2006). These results illustrate how circadian oscillators, although ubiquitously expressed, regulate gene expression and function in a cell-type-dependent manner, ensuring that within each tissue, relevant pathways are appropriately controlled (e.g., gonadal steroid hormones in reproductive tissues and glucose, and lipid metabolism in metabolic tissues).

The ER-Per2 interaction is not the first case where core clock factors have been shown to interact directly with members of the nuclear receptor family in peripheral tissues. Clock was found to interact in a hormone-dependent manner with some nuclear receptors, including RAR $\alpha$ and RXR $\alpha$. These interactions negatively regulate circadian-mediated transcriptional activation in vascular cells (McNamara et al. 2001). Another example of cross-talk between the circadian transcriptional system and nuclear receptors was recently reported in metabolically active tissues. A large number of nuclear receptors ( 28 of the 49 mouse nuclear receptors) were found to display tissuespecific oscillation in liver, skeletal muscle, and adipose tissues, providing an explanation for the well-recognized role of the circadian clock in energy balance and general metabolism. The nuclear receptors could provide a global network of proteins under circadian regulation through which environmental signals can be integrated to regulate endocrine and metabolic physiology (X. Yang et al. 2006). This network of proteins could also provide a route by which circadian control influences susceptibility to cancer development. 


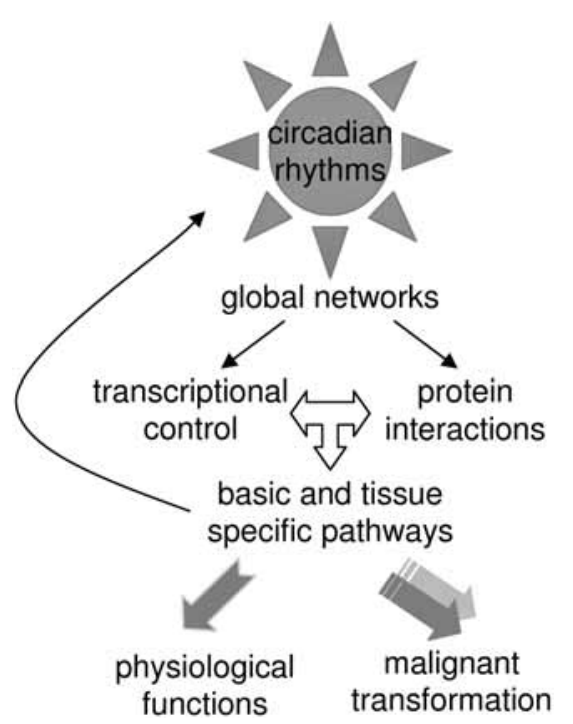

Figure 1. The circadian clock controls normal physiology and is associated with malignant transformation. Circadian clocks are found in most cells where they influence pathways critical to cell growth and survival, as well as tissue-specific functions through the control of gene expression and protein interactions. Genomewide mechanisms such as remodeling of chromatin and links between clock components and superprotein families (like checkpoint proteins and nuclear receptors) may act in concert with systemic circadian regulation to communicate signals to numerous physiological processes. Disruption of this extensive system may contribute to tumorigenesis and cancer progression in more than one way.

\section{CONCLUSION}

The circadian system integrates environmental and endogenous signals; subsequently, by means of transcriptional regulation and protein interactions, circadian oscillators relay this information to fundamental as well as tissue-specific cellular pathways to synchronize the physiology and behavior of the organism (Fig. 1). A convergence of data demonstrate the pervasive role circadian regulation has on almost every aspect of normal cellular physiology, as well as many aspects of malignant transformation. As we learn more about the molecular workings of this complex system, the implications for disruption of circadian rhythms, often associated with modern lifestyle, become apparent. The extent to which circadian regulation contributes to carcinogenesis and the ways by which core clock factors modify gene expression and protein function require further exploration. Even so, far-reaching therapeutic strategies include the use of clock factors as new targets for pharmaceutical compounds and for chronotherapy as a way to optimize the efficacy of current therapies.

\section{REFERENCES}

Bartsch C., Bartsch H., Fluchter S.H., Attanasio A., and Gupta D. 1985. Evidence for modulation of melatonin secretion in men with benign and malignant tumors of the prostate: Relationship with the pituitary hormones. J. Pineal Res. 2: 121.

Chen S.T., Choo K.B., Hou M.F., Yeh K.T., Kuo S.J., and Chang
J.G. 2005. Deregulated expression of the PER1, PER2 and PER3 genes in breast cancers. Carcinogenesis 26: 1241.

Collis S.J., Barber L.J., Clark A.J., Martin J.S., Ward J.D., and Boulton S.J. 2007. HCLK2 is essential for the mammalian Sphase checkpoint and impacts on Chk1 stability. Nat. Cell Biol. 9: 391.

Crosio C., Cermakian N., Allis C.D., and Sassone-Corsi P. 2000. Light induces chromatin modification in cells of the mammalian circadian clock. Nat. Neurosci. 3: 1241.

Curtis A.M., Seo S.B., Westgate E.J., Rudic R.D., Smyth E.M., Chakravarti D., FitzGerald G.A., and McNamara P. 2004. Histone acetyltransferase-dependent chromatin remodeling and the vascular clock. J. Biol. Chem. 279: 7091.

Doi M., Hirayama J., and Sassone-Corsi P. 2006. Circadian regulator CLOCK is a histone acetyltransferase. Cell 125: 497.

Etchegaray J.P., Lee C., Wade P.A., and Reppert S.M. 2003. Rhythmic histone acetylation underlies transcription in the mammalian circadian clock. Nature 421: 177.

Fahrenkrug J., Georg B., Hannibal J., Hindersson P., and Gras S. 2006. Diurnal rhythmicity of the clock genes Per1 and Per2 in the rat ovary. Endocrinology 147: 3769.

Filipski E., Li X.M., and Levi F. 2006. Disruption of circadian coordination and malignant growth. Cancer Causes Control 17: 509 .

Fu L., Pelicano H., Liu J., Huang P., and Lee C. 2002. The circadian gene Period2 plays an important role in tumor suppression and DNA damage response in vivo. Cell 111: 41.

Gery S., Virk R., Chumakov K., Yu A., and Koeffler H.P. 2007 a. The clock gene Per2 links the circadian system to the estrogen receptor. Oncogene. 2007. Jun 18; [Epub ahead of print].

Gery S., Gombart A.F., Yi W.S., Koeffler C., Hofmann W.K., and Koeffler H.P. 2005. Transcription profiling of C/EBP targets identifies Per2 as a gene implicated in myeloid leukemia. Blood 106: 2827

Gery S., Komatsu N., Baldjyan L., Yu A., Koo D., and Koeffler H.P. 2006. The circadian gene per1 plays an important role in cell growth and DNA damage control in human cancer cells. Mol. Cell 22: 375

Gery S., Komatsu N., Kawamata N., Miller C.W., Desmond J., Virk R.K., Marchevsky A., McKenna R., Taguchi H., and Koeffler H.P. 2007b. Epigenetic silencing of the candidate tumor suppressor gene Per1 in non-small cell lung cancer. Clin. Cancer Res. 13: 1399

Granda T.G., Liu X.H., Smaaland R., Cermakian N., Filipski E., Sassone-Corsi P., and Levi F. 2005. Circadian regulation of cell cycle and apoptosis proteins in mouse bone marrow and tumor. FASEB J. 19: 304.

Hansen J. 2001. Light at night, shiftwork, and breast cancer risk. J. Natl. Cancer Inst. 93: 1513.

Hua H., Wang Y., Wan C., Liu Y., Zhu B., Yang C., Wang X., Wang Z., Cornelissen-Guillaume G., and Halberg F. 2006. Circadian gene mPer2 overexpression induces cancer cell apoptosis. Cancer Sci. 97: 589.

Hunt T. and Sassone-Corsi P. 2007. Riding tandem: Circadian clocks and the cell cycle. Cell 129: 461.

Jones P.A. and Baylin S.B. 2007. The epigenomics of cancer. Cell 128: 683.

Ko C.H. and Takahashi J.S. 2006. Molecular components of the mammalian circadian clock. Hum. Mol. Genet. 15: R271.

Lee C.C. 2006. Tumor suppression by the mammalian Period genes. Cancer Causes Control 17: 525.

Lowrey P.L. and Takahashi J.S. 2004. Mammalian circadian biology: Elucidating genome-wide levels of temporal organization. Annu. Rev. Genomics Hum. Genet. 5: 407.

Matsuo T., Yamaguchi S., Mitsui S., Emi A., Shimoda F., and Okamura H. 2003. Control mechanism of the circadian clock for timing of cell division in vivo. Science 302: 255.

McDonnell D.P. and Norris J.D. 2002. Connections and regulation of the human estrogen receptor. Science 296: 1642.

McNamara P., Seo S.P., Rudic R.D., Sehgal A., Chakravarti D., and FitzGerald G.A. 2001. Regulation of CLOCK and MOP4 by nuclear hormone receptors in the vasculature: A humoral mechanism to reset a peripheral clock. Cell 105: 877.

Metz R.P., Qu X., Laffin B., Earnest D., and Porter W.W. 2006. 
Circadian clock and cell cycle gene expression in mouse mammary epithelial cells and in the developing mouse mammary gland. Dev. Dyn. 235: 263.

Miller B.H., McDearmon E.L., Panda S., Hayes K.R., Zhang J., Andrews J.L., Antoch M.P., Walker J.R., Esser K.A., Hogenesch J.B., and Takahashi J.S. 2007. Circadian and CLOCK-controlled regulation of the mouse transcriptome and cell proliferation. Proc. Natl. Acad. Sci. 104: 3342.

Nagoshi E., Saini C., Bauer C., Laroche T., Naef F., and Schibler U. 2004. Circadian gene expression in individual fibroblasts: Cell-autonomous and self-sustained oscillators pass time to daughter cells. Cell 119: 693

Nakamura T.J., Moriya T., Inoue S., Shimazoe T., Watanabe S., Ebihara S., and Shinohara K. 2005. Estrogen differentially regulates expression of Per1 and Per2 genes between central and peripheral clocks and between reproductive and nonreproductive tissues in female rats. J. Neurosci. Res. 82: 622.

Naruse Y., Oh-hashi K., Iijima N., Naruse M., Yoshioka H., and Tanaka M. 2004. Circadian and light-induced transcription of clock gene Per1 depends on histone acetylation and deacetylation. Mol. Cell. Biol. 24: 6278.

Panda S., Antoch M.P., Miller B.H., Su A.I., Schook A.B., Straume M., Schultz P.G., Kay S.A., Takahashi J.S., and Hogenesch J.B. 2002. Coordinated transcription of key pathways in the mouse by the circadian clock. Cell 109: 307 .

Perrin J.S., Segall L.A., Harbour V.L., Woodside B., and Amir S. 2006. The expression of the clock protein PER2 in the limbic forebrain is modulated by the estrous cycle. Proc. Natl. Acad. Sci. 103: 5591.

Pogue-Geile K.L., Lyons-Weiler J., and Whitcomb D.C. 2006. Molecular overlap of fly circadian rhythms and human pancreatic cancer. Cancer Lett. 243: 55.

Pregueiro A.M., Liu Q., Baker C.L., Dunlap J.C., and Loros J.J. 2006. The Neurospora checkpoint kinase 2: A regulatory link between the circadian and cell cycles. Science 4: 313 .

Ravindra T., Lakshmi N.K., and Ahuja Y.R. 2006. Melatonin in pathogenesis and therapy of cancer. Indian J. Med. Sci. 60: 523.

Reddy A.B., Wong G.K., O'Neill J., Maywood E.S., and Hastings M.H. 2005. Circadian clocks: Neural and peripheral pacemakers that impact upon the cell division cycle. Mutat. Res. 574: 76.

Reppert S.M. and Weaver D.R. 2002. Coordination of circadian timing in mammals. Nature 418: 935.

Ripperger J.A. and Schibler U. 2006. Rhythmic CLOCK-
BMAL1 binding to multiple E-box motifs drives circadian Dbp transcription and chromatin transitions. Nat. Genet. 38: 369 .

Schibler U. and Sassone-Corsi P. 2002. A web of circadian pacemakers. Cell 111: 919.

Shearman L.P., Sriram S., Weaver D.R., Maywood E.S., Chaves I., Zheng B., Kume K., Lee C.C., van der Horst G.T., Hastings M.H., and Reppert S.M. 2000. Interacting molecular loops in the mammalian circadian clock. Science 288: 1013.

Shiloh Y. 2003. ATM and related protein kinases: Safeguarding genome integrity. Nat. Rev. Cancer 3: 155.

Stevens R.G. 2005. Circadian disruption and breast cancer: From melatonin to clock genes. Epidemiology 16: 254.

Storch K.F., Lipan O., Leykin I., Viswanathan N., Davis F.C., Wong W.H., and Weitz C.J. 2002. Extensive and divergent circadian gene expression in liver and heart. Nature 417: 78.

Unsal-Kacmaz K., Mullen T.E., Kaufmann W.K., and Sancar A. 2005. Coupling of human circadian and cell cycles by the timeless protein. Mol. Cell. Biol. 25: 3109.

Unsal-Kacmaz K., Chastain P.D., Qu P.P., Minoo P., CordeiroStone M., Sancar A., and Kaufmann W.K. 2007. The human Tim/Tipin complex coordinates an Intra-S checkpoint response to UV that slows replication fork displacement. $\mathrm{Mol}$. Cell. Biol. 27: 3131 .

Woelfle M.A., Ouyang Y., Phanvijhitsiri K., and Johnson C.H. 2004. The adaptive value of circadian clocks: An experimental assessment in cyanobacteria. Curr. Biol. 14: 1481.

Yager J.D. and Davidson N.E. 2006. Estrogen carcinogenesis in breast cancer. N. Engl. J. Med. 354: 270.

Yang M.Y., Chang J.G., Lin P.M., Tang K.P., Chen Y.H., Lin H.Y., Liu T.C., Hsiao H.H., Liu Y.C., and Lin S.F. 2006. Downregulation of circadian clock genes in chronic myeloid leukemia: Alternative methylation pattern of hPER3. Cancer Sci. 97: 1298

Yang X., Downes M., Yu R.T., Bookout A.L., He W., Straume M., Mangelsdorf D.J., and Evans R.M. 2006. Nuclear receptor expression links the circadian clock to metabolism. Cell 126: 801 .

Yeh K.T., Yang M.Y., Liu T.C., Chen J.C., Chan W.L., Lin S.F., and Chang J.G. 2005. Abnormal expression of period 1 (PER1) in endometrial carcinoma. J. Pathol. 206: 111.

Yoshizawa-Sugata N. and Masai H. 2007. Human Tim/ Timeless-interacting protein, Tipin, is required for efficient progression of S phase and DNA replication checkpoint. $J$. Biol. Chem. 282: 2729. 


\section{$8_{\mathrm{CSH}}^{\infty} \mathrm{CH}$ Cold Spring Harbor Symposia SYMPOSIA}

\section{The Role of Circadian Regulation in Cancer}

S. Gery and H. P. Koeffler

Cold Spring Harb Symp Quant Biol 2007 72: 459-464

Access the most recent version at doi:10.1101/sqb.2007.72.004

References This article cites 50 articles, 12 of which can be accessed free at: http://symposium.cshlp.org/content/72/459.full.html\#ref-list-1

\section{License}

Email Alerting

Receive free email alerts when new articles cite this article - sign up in the box at the Service top right corner of the article or click here.

To subscribe to Cold Spring Harbor Symposia on Quantitative Biology go to: http://symposium.cshlp.org/subscriptions 